\title{
Improving the optical efficiency of white light-emitting diodes based on phosphor-in-glass by a dual-layer remote phosphorus structure with the application of $\mathrm{LiLaO}_{2}: \mathrm{Eu}^{3+}$ and $\mathrm{CaSO}_{4}: \mathrm{Ce}^{3+}$, $\mathrm{Mn}^{2+}$
}

\author{
Phan Xuan Le, Le Hung Tien \\ Faculty of Engineering, Van Lang University, Vietnam
}

\begin{tabular}{|c|c|}
\hline Article Info & ABSTRACT \\
\hline Article history: & While the remote phosphor structure is not an appropriate solution for white \\
\hline & light emitting diode (WLED) color uniformity, it is more advantageous for the \\
\hline Received Feb 23, 2021 & luminous output of WLED than the conformal phosphor or in-cup phosphor \\
\hline Revised Apr 26, 2021 & structures. Acknowledging the ability of the remote phosphor structure, many \\
\hline Accepted May 25, 2021 & $\begin{array}{l}\text { studies have been carried out to surmount the color quality disadvantage of } \\
\text { this structure. A dual-layer remote phosphor configuration is proposed in this }\end{array}$ \\
\hline Keywords: & $\begin{array}{l}\text { research paper to acquire better color quality for WLEDs through heightening } \\
\text { the color rendering index (CRI) and the color quality scale (CQS). The color }\end{array}$ \\
\hline $\mathrm{CaSO}_{4}: \mathrm{Ce}^{3+}, \mathrm{Mn}^{2+}$ & temperature of the WLED packages this study is $8500 \mathrm{~K}$. By inserting a layer \\
\hline Color quality & YAG:Ce ${ }^{3+}$ phosphor layer, the phosphor structure configuration can be \\
\hline $\mathrm{LiLaO}_{2}: \mathrm{Eu}^{3+}$ & constructed. Then, to get the best color quality, the concentration of added \\
\hline Lumen output & phosphor $\mathrm{LiLaO}_{2}: \mathrm{Eu}^{3+}$ would be changed. The findings showed the rise of \\
\hline Mie-scattering theory & CRI and CQS along with the $\mathrm{LiLaO}_{2}: \mathrm{Eu}^{3+}$, which implies the influence of \\
\hline WLEDs & $\begin{array}{l}\mathrm{LiLaO}_{2}: \mathrm{Eu}^{3+} \text { to the growth of red light components within WLEDs packages. } \\
\text { The greater the concentration of } \mathrm{LiLaO}_{2}: \mathrm{Eu}^{3+} \text { is, the more the CRI and CQS } \\
\text { increase. Meanwhile, the luminous flux gains from the green phosphor } \\
\mathrm{CaSO}_{4}: \mathrm{Ce}^{3+}, \mathrm{Mn}^{2+} \text {. }\end{array}$ \\
\hline
\end{tabular}

This is an open access article under the CC BY-SA license.

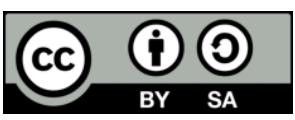

\section{Corresponding Author:}

Phan Xuan Le

Faculty of Engineering

Van Lang University

No. 45 Nguyen Khac Nhu Street, Ho Chi Minh City, Vietnam

Email: le.px@vlu.edu.vn

\section{INTRODUCTION}

As the lighting industry has higher requirements for lighting solution these days, phosphor converted white light emitting diodes (pc-WLEDs) are considered to be the fourth viable light source generation and can be an alternative to the conventional one [1]. In our daily life, the popularity of white light-emitting diodes is undeniable. In several ways, such as architecture, street lighting, backlighting, and more, they are used. However, there are two principal factors that white LEDs require enhancements to widespread their usage: the efficiency in light extraction and the angular uniformity of associated color temperatures [2]. Further breakthroughs in lumen output and color quality of WLEDs are therefore necessary for meeting high market requirements as well as life application requirements [3]. The combination of blue light from the reverse red phosphorus with yellow light from the LED chip is one of the most common methods for enhancing this potential light source. It is undeniable that the factors considered to be the determining effects 
on the efficiency of lumen output, especially the color rendering index (CRI) are the LEDs' structure and the arrangement of phosphor layers [4]-[8]. Therefore, there are several phosphor coating methods commonly utilized for LEDs' development, including dispensing and conformal coating approaches [9], [10]. However, the color quality of these structures is low due to the degraded light conversion of the phosphors. The cause of this degradation is the rise in temperature at the interface between the LED chip and phosphor layer, where the yellow emitting phosphor particle and the chip directly contact each other. Therefore, a decrease in the thermal outcome is essential to improve the efficiency of phosphor layers and prevent the irreversible destruction of phosphors. In several previous researches, it is showed that the remote phosphor structure can reduce the heat effect as the phosphor layers of this structure are placed distantly from the LED chip which is also known as a heat source. There is an appropriate gap between the layers of phosphors and the LED chip that could restrict the backscattering and light circulation within the LEDs. Therefore, this is an ideal solution for controlling the heat from the LED chip in order to substantially increase the lumen efficacy and color quality of the LEDs [11]-[16]. The remote phosphor structure can manage to meet the requirements of regular lighting but for those of other illumination applications, it cannot completely fulfil. Thus, the development of the next WLED's generation is understandable and essential. For the next upgrade LEDs packages, several new remote phosphor configurations are introduced to reduce the backscattering events and improve the luminous efficiency. In other words, the focus of using these remote phosphor models is to limit the events in which the phosphors scatter back to the chip. A research showed that the light path from the chip to the surface of a LED package can be redirected by a structure of an inverted cone lens encapsulant combining with a surrounding ring remote phosphor layer, which leads to the reduction in the light loss caused by internal reflection [17]. In addition, if a patterned remote phosphorus configuration has a clear region, which is not phosphorus-coated on the surrounding surface, in the perimeter area, high uniformity of angular-dependent associated color temperature and stability of color could be achieved [18]. Moreover, it is also useful to use patterned sapphire substrate in remote phosphorus as this pattern could increase the associated color temperature uniformity in a far-field pattern, compared to that in the original pattern [19]-[21]. The dual-layer remote phosphor structure is then proposed to increase the light performance of LED packages. Previous studies have focused on improving the color uniformity and luminous output of WLEDs when using the remote phosphor structure, but have focused only on low color temperature singlechip WLEDs. Meanwhile, it is a difficult and complicated task to improve the lighting parameters for WLEDs with a high color temperature. Thus, no previous study has shown any comparison between the efficacies of different dual-layer phosphor configurations. Thus, to optimize the color quality and luminous flux of WLEDs, it is difficult for manufacturers to determine an optimal remote phosphor layer structure.

This research paper introduces two distinct dual-layer remote phosphor structures to achieve the objective of optimizing the color consistency of WLEDs at a high color temperature of $8500 \mathrm{~K}$. The first concept of this study is the use of the green-emitting phosphor $\mathrm{CaSO}_{4}: \mathrm{Ce}^{3+}, \mathrm{Mn}^{2+}$ layer to increase the greenlight portion in WLEDs, then heightening the luminous flux. In order to increase the CRI and CQS values, the second one uses the red $\mathrm{LiLaO}_{2}: \mathrm{Eu}^{3+}$ phosphorus layer to achieve a growth in the red light component. In addition, this research includes a detailed explanation of $\mathrm{LiLaO}_{2}: \mathrm{Eu}^{3+}$ chemical's composition, which has an important impact on the lighting performances of WLEDs. Besides, the results of this research have shown that when the phosphor $\mathrm{LiLaO}_{2}: \mathrm{Eu}^{3+}$ is added, there is a noticeable improvement in the values of CRI and CQS. However, when the concentrations of blue or red phosphorus rise excessively, the concentration of $\mathrm{CaSO}_{4}: \mathrm{Ce}^{3+}, \mathrm{Mn}^{2+}$ and $\mathrm{LiLaO}_{2}: \mathrm{Eu}^{3+}$ must be modified to an appropriate amount to avoid a dramatic reduction in the quality of color or lumen output. There are three major variations in having a green or red phosphor film along with the existing YAG: $\mathrm{Ce}^{3+}$ yellow phosphor layer from the results of the studies. First, the increase of components of blue or red light will enhance the spectrum of white light, the most important point in enhancing the quality of the color. Second, depending on the concentrations of the additional phosphor layers, the scattering and the transmission of lights in WLEDs are opposite. That is why it is an important job to increase the luminous flux of WLEDs to establish an appropriate amount for phosphor layers' concentrations.

\section{DETAIL OF EXPERIMENT AND SIMULATION}

\subsection{Preparation of phosphor materials}

Before constructing the remote phosphor configurations, red and green phosphors, $\mathrm{LiLaO}_{2}: \mathrm{Eu}^{3+}$, and $\mathrm{CaSO}_{4}: \mathrm{Ce}^{3+}, \mathrm{Mn}^{2+}$, must be prepared with the ingredients having mole percent and weight listed in Table 1 and Table 2. To generate $\mathrm{LiLaO}_{2}: \mathrm{Eu}^{3+}$, it is important to strictly follow a 6-step process consisting of mixing ingredients, dehydrating, firing, powderizing, re-firing, and re-powderizing. First, slurrying all the ingredients in methanol to mix them together. After the combination become homogeneous, let it dry in air, and then grind the dry mixture into powder. Next, the powder is fired in open alumina crucibles at $600{ }^{\circ} \mathrm{C}$ with air flows. The powder now is solidified and need to be powderized before being re-fired in the same machine.

Improving the optical efficiency of white light-emitting diodes based on phosphor-in ... (Phan Xuan Le) 
For the second firing, the temperature is set at $1000^{\circ} \mathrm{C}$, and the time is 1 hour. Once the second firing process finishes, grinding the product to achieve $\mathrm{LiLaO}_{2}: \mathrm{Eu}^{3+}$ phosphor. The characteristics of $\mathrm{LiLaO}_{2}: \mathrm{Eu}^{3+}$ phosphor can be listed is being as: Emitting red emission at the emission peak from 1.775 to $2.02 \mathrm{eV}$. It is a must to put the final product in a well-closed container for a good preservation and avoiding contamination. The chemical composition of $\mathrm{LiLaO}_{2}: \mathrm{Eu}^{3+}$ green phosphor is detailed in Table 1. The preparation of $\mathrm{CaSO}_{4}: \mathrm{Ce}^{3+}$, $\mathrm{Mn}^{2+}$ consists of four firing processes in capped quartz tubes with $\mathrm{N}_{2}$, but first, the ingredients must be mixed by drying grinding or milling. After this step, the firing stages will be started. The first firing of the material will be carried out for an hour at $1000^{\circ} \mathrm{C}$. When the firing time is over, the product will be powderized. Then, it will be fired for the second time for an hour at $800^{\circ} \mathrm{C}$. When this process is finished, the product will be taken out and powderized once again. Next, it is fired for the third and fourth time at the same temperature $700^{\circ} \mathrm{C}$. The outcomes are also need to be powderized. The final product should be washed in water several times and dried for the last time. The obtained $\mathrm{CaSO}_{4}: \mathrm{Ce}^{3+}, \mathrm{Mn}^{2+}$ will have emission color of yellow-green and emission peak at $2.35 \mathrm{eV}$.

Table 1. Ingredients of $\mathrm{LiLaO}_{2}: \mathrm{Eu}^{3+}$ phosphor composition

\begin{tabular}{ccc}
\hline Ingredient & Mole \% & By weight $(\mathrm{g})$ \\
\hline $\mathrm{La}_{2} \mathrm{O}_{3}$ & 95 (of La) & 155 \\
$\mathrm{Eu}_{2} \mathrm{O}_{3}$ & 5 (of Eu) & 8.8 \\
$\mathrm{Li}_{2} \mathrm{CO}_{3}$ & 101 (of Li) & 37.4 \\
\hline
\end{tabular}

Table 2. Ingredients of $\mathrm{CaSO}_{4}: \mathrm{Ce}^{3+}, \mathrm{Mn}^{2+}$ phosphor composition

\begin{tabular}{ccc}
\hline Ingredient & Mole \% & By weight $(\mathrm{g})$ \\
\hline $\mathrm{CaSO}_{4}$ & 88.5 & 121 \\
$\mathrm{CeO}_{2}$ & 2.5 & 4.3 \\
$\mathrm{MnCO}_{4}$ & 6 & 6.9 \\
$\left(\mathrm{NH}_{4}\right)_{2} \mathrm{SO}_{4}$ & $\sim 10$ & 13.2 \\
\hline
\end{tabular}

\subsection{Simulation process}

Two different dual-layer phosphor configurations, including the green-yellow configuration (GYC) and the red-yellow configuration (RYC), were presented in this article. Both the GYC and RYC structures are built on blue chips with two phosphor layers. In particular, the phosphorus layer $\mathrm{CaSO}_{4}: \mathrm{Ce}^{3+}, \mathrm{Mn}^{2+}$ is above the YAG:Ce ${ }^{3+}$ yellow phosphorus layer in the GYC structure, as shown in Figure 1 (a). In the meanwhile, the phosphorous layer of $\mathrm{LiLaO}_{2}: \mathrm{Eu}^{3+}$ is set above YAG:Ce $\mathrm{Ce}^{3+}$ yellow phosphorous layer in the RYC structure, as shown in Figure 1 (b). In Figure 1 (c), the measured spectra of the red-emitting $\mathrm{LiLaO}_{2}: \mathrm{Eu}^{3+}$ phosphor is displayed. The purpose of applying the GYC and $\mathrm{RYC}$ is to acquire the enhancement in the quality of color and lumen efficacy of WLEDs. This goal can be achieved by pushing the component of green scattering and red light inside the packages of the WLED. Nevertheless, the concentrations of $\mathrm{CaSO}_{4}: \mathrm{Ce}^{3+}, \mathrm{Mn}^{2+}$, and $\mathrm{LiLaO}_{2}: \mathrm{Eu}^{3+}$ phosphors must be adjusted accordingly to succeed in doing this. The concentrations of green $\mathrm{CaSO}_{4}: \mathrm{Ce}^{3+}, \mathrm{Mn}^{2+}$, and red $\mathrm{LiLaO}_{2}: \mathrm{Eu}^{3+}$ phosphors shift in the opposite direction to that of yellow YAG: $\mathrm{Ce}^{3+}$ phosphorus, as can be seen in Figure 2. The average CCTs will be maintained by this distinct change which will affect the scattering and absorption of phosphorus layers in WLEDs. This would undoubtedly have a great influence on the consistency of the color and the luminescence of the LED lamps. The choice of the concentration of these phosphorous $\mathrm{CaSO}_{4}: \mathrm{Ce}^{3+}, \mathrm{Mn}^{2+}$, and $\mathrm{LiLaO}_{2}: \mathrm{Eu}^{3+}$ is therefore a deciding factor for improving the color quality of the WLED. Specifically, when the concentrations of $\mathrm{CaSO}_{4}: \mathrm{Ce}^{3+}, \mathrm{Mn}^{2+}$, and $\mathrm{LiLaO}_{2}: \mathrm{Eu}^{3+}$ increase from $2 \%$ to $20 \%$ wt., YAG: $\mathrm{Ce}^{3+}$ concentration decreases in order to maintain the average CCT, even the high color temperature of the WLEDs which is $8500 \mathrm{~K}$.

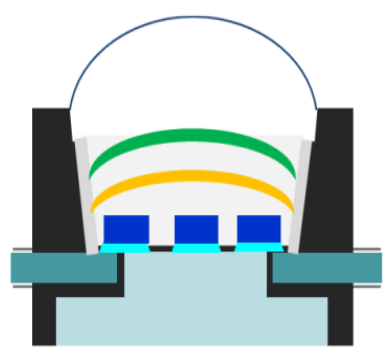

(a)

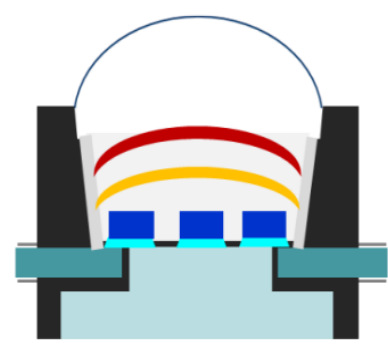

(b)

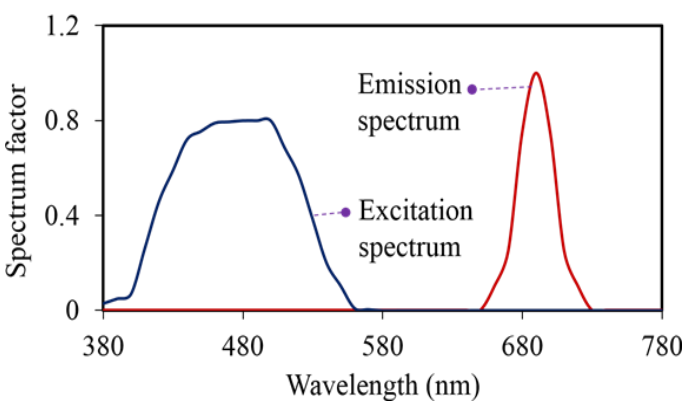

(c)

Figure 1. Illustrations of, (a) GYC, (b) RYC, (c) the measured spectra of the red-emitting $\mathrm{LiLaO}_{2}: \mathrm{Eu}^{3+}$ phosphor 


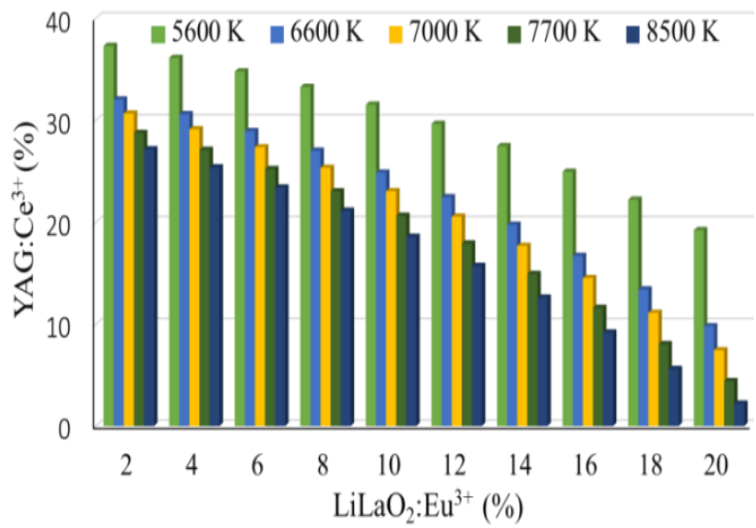

(a)

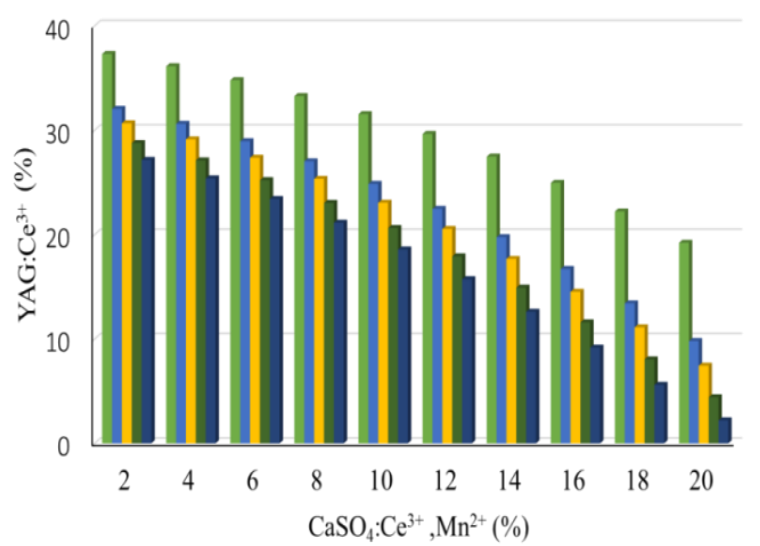

(b)

Figure 2. The change of phosphor concentration of RYC (above) and GYC (below) for keeping the average $\mathrm{CCT}$, (a) red $\mathrm{LiLaO}_{2}: \mathrm{Eu}^{3+}$, (b) green $\mathrm{CaSO}_{4}: \mathrm{Ce}^{3+}, \mathrm{Mn}^{2+}$

On top of that, as displayed in Figure 3, the effect on the WLED spectrum caused by concentration of red $\mathrm{LiLaO}_{2}: \mathrm{Eu}^{3+}$ is noticeable. The red-yellow configuration demonstrates a rise in spectral emissions in three distinct regions compared to the green phosphorus $\mathrm{CaSO}_{4}: \mathrm{Ce}^{3+}, \mathrm{Mn}^{2+}$ structure. In Figure 3(a), as the $\mathrm{CaSO}_{4}: \mathrm{Ce}^{3+}, \mathrm{Mn}^{2+}$ concentration increases, the intensity increases in the spectral regions of $420 \mathrm{~nm}-480 \mathrm{~nm}$ and $500 \mathrm{~nm}-640 \mathrm{~nm}$. In other words, the rise in the emission spectrum indicates a greater luminous flux in these two regions. In addition, there are further internal blue-light scattering events for $\mathrm{CaSO}_{4}: \mathrm{Ce}^{3+}, \mathrm{Mn}^{2+}$, which implies that the phosphor scattering within the LEDs is improved, and the copper color is benefited. Meanwhile, with the rise in the concentration of $\mathrm{LiLaO}_{2}: \mathrm{Eu}^{3+}$, the emission spectra show an improvement in $648 \mathrm{~nm}-738 \mathrm{~nm}$ region. However, without the increase emission spectra in the two similar regions of 420 $\mathrm{nm}-480 \mathrm{~nm}$ and $500 \mathrm{~nm}-640 \mathrm{~nm}$, this development is unimportant, because the increase intensity of these two spectral regions will be beneficial to the lumen output of blue light (blue-light scattering). The growth in emission spectra along with the increase of color temperature is another result that can be seen from Figure 3 (b). In other words, the higher the temperature of the color, the higher the spectral emission becomes. Hence, it is possible to achieve better the color and optical quality. This result is an essential reference for LEDs manufacture with the implementation of $\mathrm{LiLaO}_{2}: \mathrm{Eu}^{3+}$ phosphor, especially when it is difficult to control the quality of WLEDs with high temperature. In brief, the research defines that red phosphor $\mathrm{LiLaO}_{2}: \mathrm{Eu}^{3+}$ will improve the color quality of WLEDs having high color temperature $(8500 \mathrm{~K})$. Based on the requirements of manufacturers, the selection of the structure can be made. If they want to manufacture the WLEDs with high color quality, a slight decrease in luminous flux is acceptable.

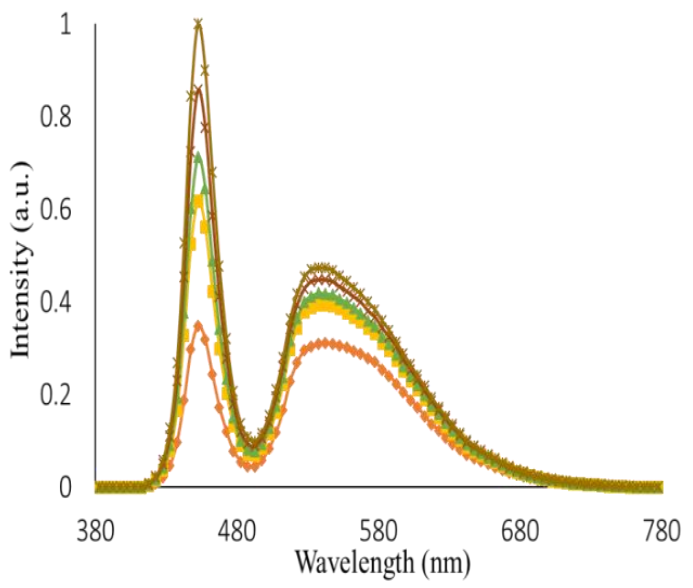

(a)

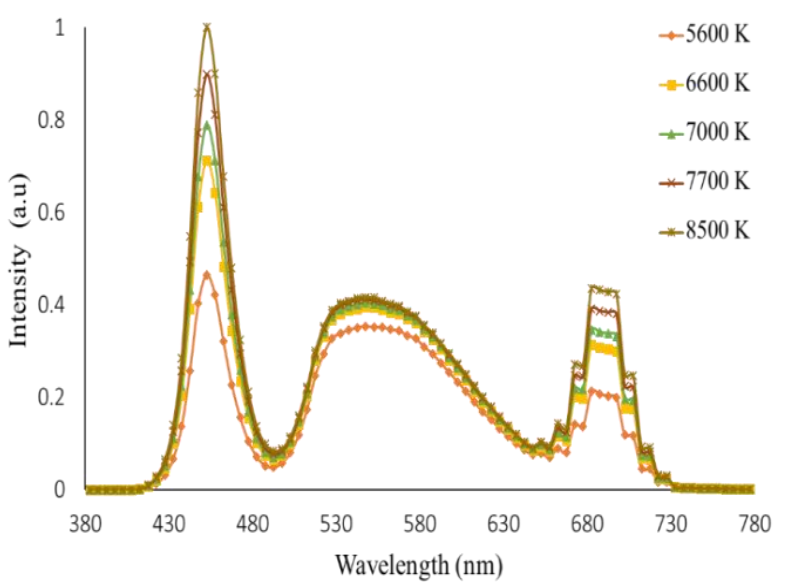

(b)

Figure 3. Emission spectra of RYC (above) and GYC (below), (a) red $\mathrm{LiLaO}_{2}: \mathrm{Eu}^{3+}$, (b) green $\mathrm{CaSO}_{4}: \mathrm{Ce}^{3+}, \mathrm{Mn}^{2+}$ 


\section{COMPUTATION AND DISCUSSION}

Compared to the real one, the color rendering index (CRI) is a measurement used to examine how the light source reveals the true color of the objects. The color imbalance occurs depending on the color principle of the three main colors green, red and yellow when the green light component is higher than the others. As a result, the WLED's light chromatic performance is negatively affected and then, reducing their color integrity. In Figure 4, when the green phosphor $\mathrm{CaSO}_{4}: \mathrm{Ce}^{3+}, \mathrm{Mn}^{2+}$ is added, the charts illustrate the decline of CRI. Specifically, CRI decreases gradually when the concentration of this phosphorus increases from $2 \%$ to $20 \%$. However, CRI is not an ideal index when being compared to color quality scale (CQS), since it does not evaluate all dimensions of the quality of illumination. These reductions in CRI are therefore acceptable when using $\mathrm{CaSO}_{4}: \mathrm{Ce}^{3+}, \mathrm{Mn}^{2+}$. It is a mixture of the three fundamental components, including CRI, individual desires, and color coordinates, in terms of CQS. Thus, CQS is a more significant objective and difficult to accomplish. According to the results from Figure 5, CQS maintains its high values while the $\mathrm{CaSO}_{4}: \mathrm{Ce}^{3+}, \mathrm{Mn}^{2+}$ concentration does not exceed $8 \%$. In comparison, the CQS declines dramatically as the $\mathrm{CaSO}_{4}: \mathrm{Ce}^{3+}, \mathrm{Mn}^{2+}$ concentration rises from $8 \%$ to $20 \%$. Therefore, an appropriately chosen concentration of $\mathrm{LiLaO}_{2}: \mathrm{Eu}^{3+}$ will fluctuate from $2 \%$ to $8 \%$ if the target is to get better color quality, after considering emitted luminous flux.

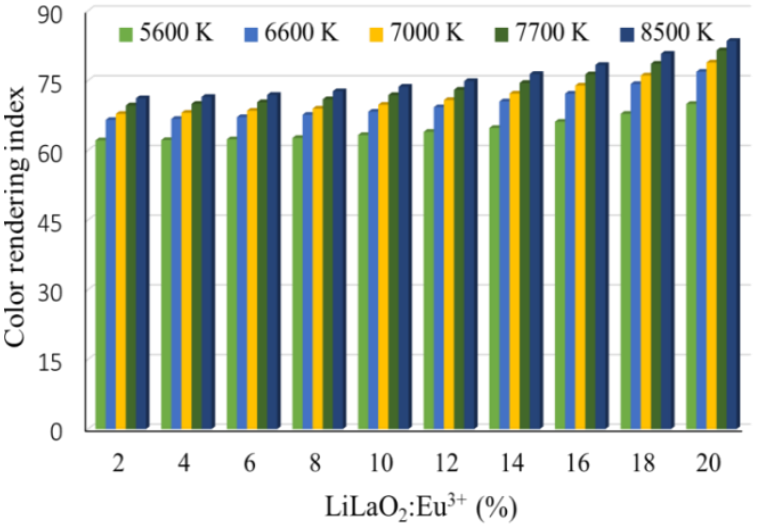

(a)

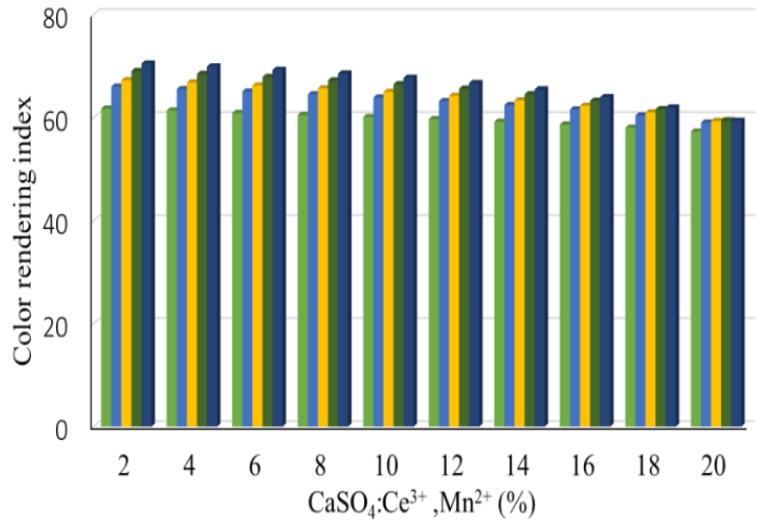

(b)

Figure 4. The color rendering index as a function of the concentration of, (a) red $\mathrm{LiLaO}_{2}: \mathrm{Eu}^{3+}$, (b) green $\mathrm{CaSO}_{4}: \mathrm{Ce}^{3+}, \mathrm{Mn}^{2+}$

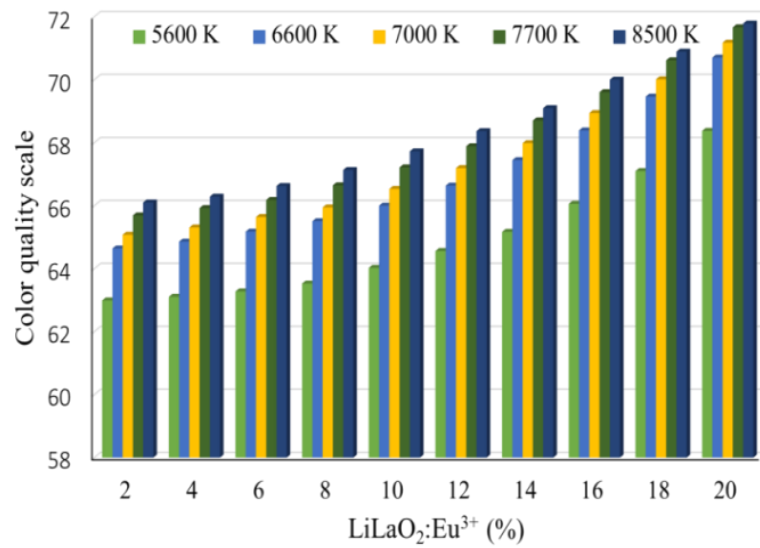

(a)

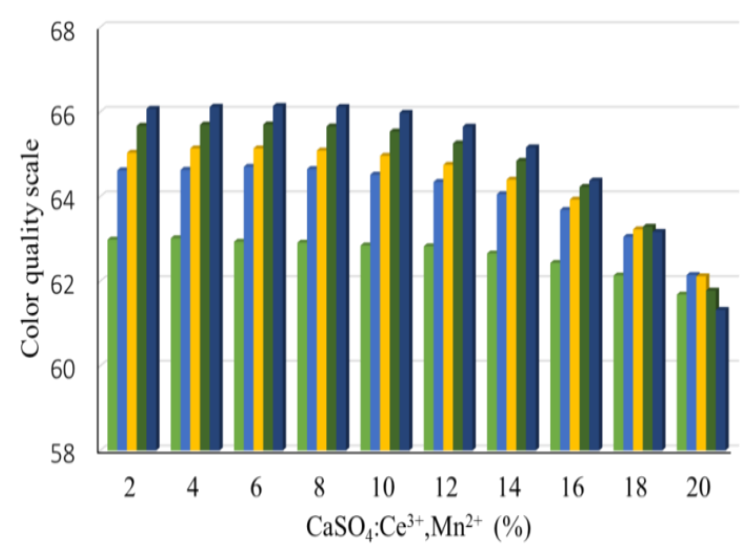

(b)

Figure 5. The color quality scale as a function of the concentration of, (a) red $\mathrm{LiLaO}_{2}: \mathrm{Eu}^{3+}$, (b) green $\mathrm{CaSO}_{4}: \mathrm{Ce}^{3+}, \mathrm{Mn}^{2+}$

In the meantime, CRI demonstrates a light rise with the presence of red phosphor $\mathrm{LiLaO}_{2}: \mathrm{Eu}^{3+}$. In other words, with the increase of $\mathrm{LiLaO}_{2}: \mathrm{Eu}^{3+}$ concentration, CRI is increased, as seen in Figure 4 (above). In 
the remote phosphor structure, the force behind this upward trend of CRI when using red phosphor $\mathrm{LiLaO}_{2}: \mathrm{Eu}^{3+}$ is its absorption feature. Not only can the blue lights from LED chips be absorbed by this red phosphor, but also the yellow light. In addition, $\mathrm{LiLaO}_{2}: \mathrm{Eu}^{3+}$ can transform the blue light into the red light while absorbing blue light. Therefore, the absorption of blue light is better than the yellow one because of its absorption feature, while this red phosphor absorbs two types of light. Consequently, the addition of $\mathrm{LiLaO}_{2}: \mathrm{Eu}^{3+}$ has increased the red light component, leading to a higher index of color rendering (CRI). For an effective modern WLED lamp, CRI is a key parameter, because if an LED package has a high color rendering index, its price would be higher than the others. However, by using $\mathrm{LiLaO}_{2}: \mathrm{Eu}^{3+}$, the cost of production can be reduced, contributing to the popularity of its use in LED manufacturing. Nevertheless, researchers have concentrated on CQS, a more synthetic index to measure the color quality of LEDs than CRI, as mentioned above. Figure 5 shows that, similar to the CRI, red phosphor $\mathrm{LiLaO}_{2}: \mathrm{Eu}^{3+}$ will boost CQS values. The first chart in Figure 5 shows that the dramatic rise in CQS is due to the increase in concentration of $\mathrm{LiLaO}_{2}: \mathrm{Eu}^{3+}$. It can therefore be concluded that adding the phosphor layer $\mathrm{LiLaO}_{2}: \mathrm{Eu}^{3+}$ can increase the quality of white light color for WLEDs built with a dual-layer phosphorus structure. This is one of the most valuable findings for manufacturers to consider when it comes to the objective of enhancing the color quality. However, it should not be ignored that $\mathrm{LiLaO}_{2}: \mathrm{Eu}^{3+}$ is somehow a downside to the lumen output of WLEDs. Next, the scientific model in which the mathematic framework of transmitted blue lights and converted yellow lights will be presented to investigate their activities in the double-layer phosphor structure. In addition, a major development in the efficacy of LED lights can be attained from this model. For a single layer remote phosphorus package with a phosphorus layer thickness of $2 h$, the transmitted blue light and converted yellow light are expressed as the two expressions below:

$$
\begin{aligned}
& P B_{1}=P B_{0} \times e^{-2 \alpha_{B 1} h} \\
& P Y_{1}=\frac{1}{2} \frac{\beta_{1} \times P B_{0}}{\alpha_{B 1}-\alpha_{Y 1}}\left(e^{-2 \alpha_{Y 1} h}-e^{-2 \alpha_{B 1} h}\right)
\end{aligned}
$$

For the dual-layer remote phosphorus structure with the phosphor layer thickness of $h$, the transmitted blue light and converted yellow light are defined as:

$$
\begin{aligned}
& P B_{2}=P B_{\mathrm{O}} \times e^{-2 \alpha_{B 2} h} \\
& P Y_{2}=\frac{1}{2} \frac{\beta_{2} \times P B_{0}}{\alpha_{B 2}-\alpha_{Y 2}}\left(e^{-2 \alpha_{Y 2} h}-e^{-2 \alpha_{B 2} h}\right)
\end{aligned}
$$

$h$ is the thickness of each coating of phosphorus. Subscripts "1" and "2" express remote phosphor structures with single and dual layers. $\beta$ indicates the conversion coefficient for the conversion of blue light into yellow light, while $\gamma$ presents the yellow light reflectance coefficient. $P B$ is the blue light intensity, and $P Y$ is the yellow light intensity. The combination of $P B$ and $P Y$ demonstrated the light intensity of the blue LED, presented by $P B_{0}$. In addition, $\alpha_{B}$ and $\alpha_{Y}$ separately describe the fractions of the blue and yellow lights' energy losses, during the light multiplication in the phosphor film, respectively. The illumination efficacy of WLEDs is higher with the double-layer phosphorus structure than with the one-layer structure:

$$
\frac{\left(P B_{2}+P Y_{2}\right)-\left(P B_{1}+P Y_{1}\right)}{P B_{1}+P Y_{1}}>0
$$

The scattering of the phosphorus particles was defined based on the Mie theory [22], [23]. Moreover, the scattering cross section of $C_{s c a}$ for spherical particles are estimated with the support from the Mie theory. Furthermore, applying the Lambert-Beer law [24], [25] can help to compute the transmitted light power:

$$
I=I o \exp \left(-\mu_{e x t} L\right)
$$

$I_{0}$ is the incident light intensity in this expression, $L$ indicates the phosphor layer's thickness (mm), and $\mu_{\text {ext }}$ is the coefficient of extinction. In addition, $\mu_{\text {ext }}$ can be calculated by: $\mu_{e x t}=N_{r}$. Cext, in which $N_{r}$ $\left(\mathrm{mm}^{-3}\right)$ is defined as the distribution of particle number density, and $\mathrm{C}_{\text {ext }}\left(\mathrm{mm}^{2}\right)$ presents the cross-section of 
phosphorus particles for extinction. Expression (5) demonstrated that the luminous efficiency of remote phosphor pc-LEDs with dual layers is higher than single layer of phosphorus. Thus, the lumen efficacy of the dual-layer remote phosphor layer added in LED packages has been carried out and proved by this study.

In Figure 6, as $\mathrm{CaSO}_{4}: \mathrm{Ce}^{3+}, \mathrm{Mn}^{2+}$ is raised from $2 \%$ wt. up to $20 \%$ wt., we can observe the development of luminous flux. In comparison, the luminous flux of the dual-layer remote phosphor structure shows a sharp decline with the rise in $\mathrm{LiLaO}_{2}: \mathrm{Eu}^{3+}$ phosphor concentration. The reduction factor $\mu_{\text {ext }}$ is obviously in direct proportion to the $\mathrm{LiLaO}_{2}: \mathrm{Eu}^{3+}$ concentration, according to the Lambert-Beer law, though in inverse proportion to the energy of light transmission. Therefore, if the thicknesses of both phosphor films are fixed, as the concentration of $\mathrm{LiLaO}_{2}: \mathrm{Eu}^{3+}$ increases, the emitted photoluminescence could decrease. However, the dual-layer phosphor structure with the additional red $\mathrm{LiLaO}_{2}: \mathrm{Eu}^{3+}$ phosphor yields better luminous flux than the single-layer phosphor structure does, as demonstrated through the calculations above. What's more, the CRI and CQS values also gain from this red phosphor. Therefore, by using $\mathrm{LiLaO}_{2}: \mathrm{Eu}^{3+}$, these benefits are obtained and this reduction in lumen output is entirely acceptable. Based on the objective they wish to achieve manufacturers will select an appropriate concentration of $\mathrm{LiLaO}_{2}: \mathrm{Eu}^{3+}$ to apply for WLEDs mass production.

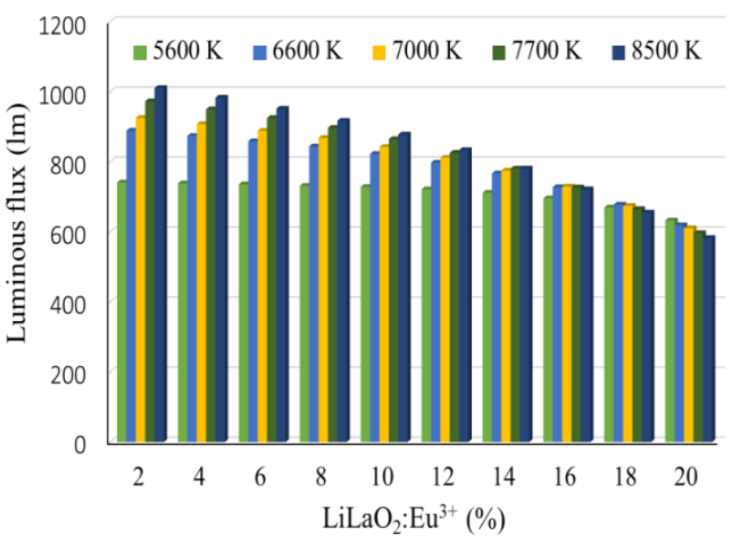

(a)

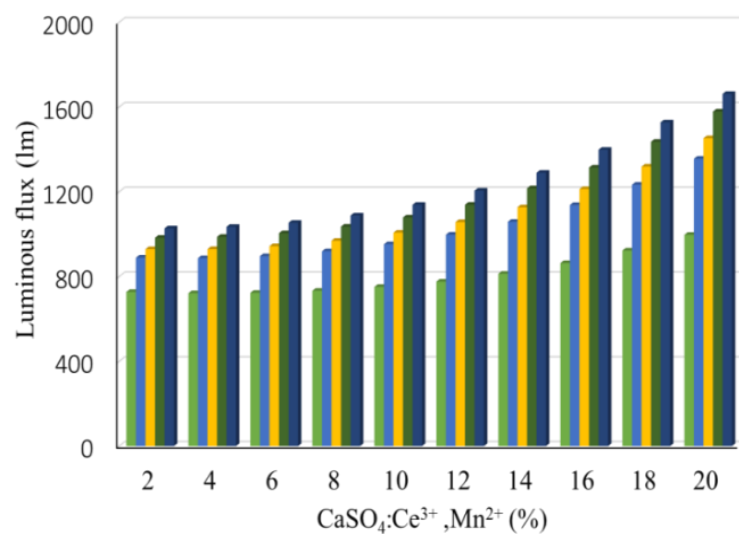

(b)

Figure 6. The lumen output as a function of the concentration of, (a) red $\mathrm{LiLaO}_{2}: \mathrm{Eu}^{3+}$, (b) green $\mathrm{CaSO}_{4}: \mathrm{Ce}^{3+}$, $\mathrm{Mn}^{2+}$

\section{CONCLUSION}

The effects of the green $\mathrm{CaSO}_{4}: \mathrm{Ce}^{3+}, \mathrm{Mn}^{2+}$ and red $\mathrm{LiLaO}_{2}: \mathrm{Eu}^{3+}$ phosphors in the dual-layer phosphor configurations on the optical features of WLEDs, including CRI, CQS and luminous flux, are shown in this study. By applying the Mie-scattering theory and the law of Lambert-Beer, it can be inferred from the study that $\mathrm{LiLaO}_{2}: \mathrm{Eu}^{3+}$ is a suitable phosphor material for improving color quality, whereas $\mathrm{CaSO}_{4}: \mathrm{Ce}^{3+}, \mathrm{Mn}^{2+}$ is the perfect choice for WLEDs to increase luminous flux. This result is not only true for low color temperature WLEDs, but also for those with a high $8500 \mathrm{~K}$ color temperature. This study has also accomplished the objective of enhancing the white-light chromatic quality that the remote phosphor structure has been struggling to acquire. A slight drawback in terms of luminous flux, however, still exists: when $\mathrm{CaSO}_{4}: \mathrm{Ce}^{3+}, \mathrm{Mn}^{2+}$ or $\mathrm{LiLaO}_{2}: \mathrm{Eu}^{3+}$ concentrations increase excessively, the quality of color or luminous flux tends to decrease dramatically. Therefore, it is very important to determine the required phosphorus concentration based on what the manufacturers aim to achieve. In addition, the results obtained from the research paper can be used as a crucial reference in the development of WLED packages of higher quality.

\section{REFERENCES}

[1] Z. Jia, et al., "Strategies to approach high performance in $\mathrm{Cr}^{3+}$-doped phosphors for high-power NIR-LED light sources," Light: Science \& Applications, vol. 9, no. 86, pp. 1-9, 2020, doi: 10.1038/s41377-020-0326-8.

[2] Q. Yao, et al., "YAG:Ce ${ }^{3+}$ transparent ceramic phosphors brighten the nextgeneration laser-driven lighting Advanced Materials, vol. 32, no. 19, p. 1907888, March 2020, doi: 10.1002/adma.201907888.

[3] G. Tan, Y. Huang, Ming-Chun Li, Seok-Lyul Lee, and Shin-Tson Wu, "High dynamic range liquid crystal displays with a mini-LED backlight," Optics Express, vol. 26, no. 13, pp. 16572-16584, 2018, doi: 10.1364/OE.26.016572. 
[4] W. Wang and P. Zhu, "Red photoluminescent Eu3+-doped Y2O3 nanospheres for LED-phosphor applications: Synthesis and characterization," Optics Express, vol. 26, pp. 34820-34829, 2018, doi: 10.1364/OE.26.034820.

[5] Chen, et al., "Flexible/curved backlight module with quantum-dots microstructure array for liquid crystal displays," Optics Express, vol. 26, no. 3, pp. 3466-3482, 2018, doi: 10.1364/OE.26.003466.

[6] Z. Li, Y. Tang, J. Li, X. Ding, C. Yan, and B. Yu, "Effect of flip-chip height on the optical performance of conformal white-light-emitting diodes," Optics Letters, vol. 43, no. 5, pp. 1015-1018, 2018, doi: 10.1364/OL.43.001015.

[7] Yu-Fang Huang, Yu-Chieh Chi, Mu-Ku Chen, Din-Ping Tsai, Ding-Wei Huang, and Gong-Ru Lin, "Red/green/blue LD mixed white-light communication at $6500 \mathrm{~K}$ with divergent diffuser optimization," Optics Express, vol. 26, no. 18, pp. 23397-23410, 2018, doi: 10.1364/OE.26.023397.

[8] S. Bindai, K. Annapurna, and A. Tarafder, "Realization of phosphor-in-glass thin film on soda-lime silicate glass with low sintering temperature for high color rendering white LEDs," Applied Optics, vol. 58, no. 9, pp. 2372-2381, 2019, doi: 10.1364/AO.58.002372.

[9] B. K. Tsai, C. C. Cooksey, D. W. Allen, C. C. White, E. Byrd, and D. Jacobs, "Exposure study on UV-induced degradation of PTFE and ceramic optical diffusers," Applied Optics, vol. 58, no. 5, pp. 1215-1222, 2019, doi: 10.1364/AO.58.001215.

[10] I. G. Palchikova, E. S. Smirnov, and E. Iv. Palchikov, "Quantization noise as a determinant for color thresholds in machine vision," Journal of the Optical Society of America A, vol. 35, no. 4, pp. B214-B222, 2018, doi: 10.1364/JOSAA.35.00B214.

[11] H. Lee, et al., "Color-tunable organic light-emitting diodes with vertically stacked blue, green, and red colors for lighting and display applications," Optics Express, vol. 26, no. 14, pp. 18351-18361, July 2018, doi: 10.1364/OE.26.018351.

[12] J. Ji, G. Zhang, S. Yang, X. Feng, X. Zhang, and C. C. Yang, "Theoretical analysis of a white-light LED array based on a GaN nanorod structure," Applied Optics, vol. 59, no. 8, pp. 2345-2351, 2020, doi: 10.1364/AO.387059.

[13] B. Zhang, et al., "Rapid, large-scale stimulated Raman histology with strip mosaicing and dual-phase detection," Biomedical Optics Express, vol. 9, no. 6, pp. 2604-2613, 2018, doi: 10.1364/BOE.9.002604.

[14] C. Bai, et al., "Full-color optically-sectioned imaging by wide-field microscopy via deep-learning," Biomedical Optics Express, vol. 11, no. 5, pp. 2619-2632, 2020, doi: 10.1364/BOE.389852.

[15] Y. Wu, et al., "Monolithic integration of MoS2-based visible detectors and GaN-based UV detectors," Photonics Research, vol. 7, no. 10, pp. 1127-1133, 2019, doi: 10.1364/PRJ.7.001127.

[16] M. Royer, "Evaluating tradeoffs between energy efficiency and color rendition," OSA Continuum, vol. 2, no. 8, pp. 2308-2327, 2019, doi: 10.1364/OSAC.2.002308.

[17] Agathi-Anthoula Kaminari, S. C. Boyatzis, and A. Alexopoulou, "Linking Infrared Spectra of Laboratory Iron Gall Inks Based on Traditional Recipes with their Material Components," Applied Spectroscopy, vol. 72, no. 10, pp. 1511-1527, 2018

[18] L. Xu, B. Zhao, and M. R. Luo, "Color gamut mapping between small and large color gamuts: Part II. gamut extension," Optics Express, vol. 26, no. 13, pp. 17335-17349, 2018, doi: 10.1364/OE.26.017335.

[19] C. Zhang, T. Han, S. Cao, X. Cheng, and J. Zhang, "Mn ${ }^{4+}$-doped fluoride phosphors rapidly synthesized by ball milling," Optical Materials Express, vol. 8, no. 1, pp. 73-81, 2018, doi: 10.1364/OME.8.000073.

[20] B. Li, et al., "High-efficiency cubic-phased blue-emitting Ba3Lu2B6O15:Ce3+ phosphors for ultraviolet-excited white-light-emitting diodes," Optics Letters, vol. 43, no. 20, pp. 5138-5141, 2018, doi: 10.1364/OL.43.005138.

[21] Ping-Ping Li, et al., "Unveiling of control on the polarization of supercontinuum spectra based on ultrafast birefringence induced by filamentation," Journal of the Optical Society of America B, vol. 35, no. 11, pp. 29162922, 2018, doi: 10.1364/JOSAB.35.002916.

[22] X. Wang, Y. Wang, J. Yu, Y. Bu, and X. Yan, "Modifying phase, shape and optical thermometry of $\mathrm{NaGdF} 4: 2 \% \mathrm{Er}^{3+}$ phosphors through $\mathrm{Ca}^{2+}$ doping," Optics Express, vol. 26, no. 17, pp. 21950-21959, 2018, doi: 10.1364/OE.26.021950

[23] S, Kumar, S. Kumar, M. Mahadevappa, and P. K. Dutta, "Extended light-source-based lensless microscopy using constrained and regularized reconstruction," Applied Optics, vol. 58, no. 3, pp. 509-516, 2019, doi: 10.1364/AO.58.000509.

[24] J. Ruschel, et al., "Current-induced degradation and lifetime prediction of $310 \mathrm{~nm}$ ultraviolet light-emitting diodes," Photonics Research, vol. 7, no. 7, pp. B36-B40, 2019, doi: 10.1364/PRJ.7.000B36.

[25] L. V. Labunets, A. B. Borzov, and I. M. Akhmetov, "Regularized parametric model of the angular distribution of the brightness factor of a rough surface," Journal of Optical Technology, vol. 86, no. 10, pp. 618-626, 2019, doi: 10.1364/JOT.86.000618. 